\title{
Cosmic ray acceleration and gamma-ray emission from protostellar jets
}

\author{
Anabella Araudo, ${ }^{a, b, c, *}$ Marco Padovani ${ }^{d}$ and Alexandre Marcowith ${ }^{a}$ \\ ${ }^{a}$ Laboratoire Univers et Particules de Montpellier (LUPM) Université Montpellier, CNRS/IN2P3, \\ CC72, place Eugène Bataillon,34095 \\ ${ }^{b}$ ELI Beamlines, Institute of Physics, Academy of Sciences, \\ 25241 Dolní Břežany, Czech Republic \\ ${ }^{c}$ Astronomical Institute, Academy of Sciences, \\ Boční II 1401, CZ-141 00 Prague, Czech Republic \\ ${ }^{d}$ INAF-Osservatorio Astrofisico di Arcetri \\ Largo E. Fermi, 5 - 50125 Firenze, Italy \\ E-mail: anabella.araudo@eli-beams.eu
}

The detection of synchrotron radio emission from jets powered by massive protostars indicates the presence of relativistic electrons and magnetic fields of strength $\sim 0.3-5 \mathrm{mG}$. We study diffusive shock acceleration and magnetic field amplification in protostellar jets with speed $1000 \mathrm{~km} \mathrm{~s}^{-1}$. We show that the magnetic field in the synchrotron emitter can be amplified by the non-resonant hybrid (Bell) instability excited by the cosmic-ray streaming. By combining the synchrotron data with basic theory of Bell instability we estimate the magnetic field in the synchrotron emitter and the maximum energy of protons. Protons can achieve maximum energies $\sim 0.1 \mathrm{TeV}$ and emit $\gamma$ rays in their interaction with matter fields. We predict detectable levels of $\gamma$ rays in IRAS 16547-5247 and IRAS 16848-4603. The detection of this radiation by the Fermi satellite in the $\mathrm{GeV}$ domain and the forthcoming Cherenkov Telescope Array at higher energies may open a new window to study the formation of massive stars, as well as diffusive acceleration and magnetic field amplification in shocks with velocities of about $1000 \mathrm{~km} \mathrm{~s}^{-1}$.

$37^{\text {th }}$ International Cosmic Ray Conference (ICRC 2021)

July 12th - 23rd, 2021

Online - Berlin, Germany

\footnotetext{
*Presenter
} 


\section{Introduction}

Stars are formed within dense molecular clouds, accreting matter onto the central protostar with the formation of a circumstellar disc and bipolar jets moving with speeds $v_{\mathrm{j}} \sim 1000 \mathrm{~km} \mathrm{~s}^{-1}$. Nonthermal radio emission has been detected in the termination region of several protostellar jets [1]. The detection of synchrotron radiation is an evidence that there is a population of mildly-relativistic or relativistic electrons in the jet, and strong magnetic fields.

- Magnetic fields in the synchrotron emitter in protostellar jets have strengths $B_{\mathrm{s}} \approx 0.1-10 \mathrm{mG}$ (see Sect. 3). These values are larger than the expected magnetic field in the jet termination region, $B_{\mathrm{j}} \approx 1-100 \mu \mathrm{G}$, requiring amplification of the magnetic field, given that compression by the shock does not produce strong enough fields.

- The energy density in non-thermal electrons and protons inferred from the synchrotron data is $\sim 10^{-8} \mathrm{erg} \mathrm{cm}^{-3}$ (see Sect. 3). The cosmic-ray (CR) energy density in molecular clouds is poorly known, but it should be at least of the same order of the CR energy density in the interstellar medium, i.e. $\sim 10^{-12} \mathrm{erg} \mathrm{cm}^{-3}$ [2]. Therefore, relativistic electrons and protons in the protostellar jets non-thermal lobes should be locally accelerated in the jet termination shocks.

In the present contribution we consider that show that the magnetic field in the synchrotron emitter of high-mass protostellar jets can be amplified by the streaming of CRs [3], as in supernova remnants [4] and jets in Active Galactic Nuclei [5]. By assuming that the number of non-thermal electrons and protons is the same, we find that the energy density in non-thermal protons is large enough to drive the Bell instability in the termination region of jets powered by the massive protostars IRAS 16547-4247 and 16484-4603. See [6] for a larger sample of sources, and a more detailed analysis.

If the non-resonant streaming instability is important and the magnetic field is amplified, the maximum energy of protons is determined by the amount of protons that escape from the shock upstream region [7, 8], giving maximum energies $\sim 0.1 \mathrm{TeV}$. Electrons diffusing in the turbulence generated by protons can achieve similar maximum energies. We model the gamma-ray emission and we show that detectable levels of gamma rays in the $\mathrm{GeV}$ domain are expected from the non-thermal lobes in the case studies.

\section{The jet termination region}

In the jet termination region, where the jet impacts against the ambient medium, a double shock structure (i.e. a working surface) is formed. In the particular case of light jets, where the jet $\left(n_{\mathrm{j}}\right)$ to molecular cloud $\left(n_{\mathrm{mc}}\right)$ density contrast is $\chi \equiv n_{\mathrm{j}} / n_{\mathrm{mc}} \ll 1$, the bow shock moves into the molecular cloud at a speed $v_{\mathrm{bs}} \ll v_{\mathrm{j}}$, and the reverse shock in the jet moves at $v_{\mathrm{rs}} \sim v_{\mathrm{j}}$. We consider that synchrotron emitting electrons are accelerated in the reverse shock with velocity $v_{\mathrm{rs}}=1000 \mathrm{~km} \mathrm{~s}^{-1}$. The condition for the reverse shock to be adiabatic can be written as

$$
\frac{v_{\mathrm{rs}}}{\mathrm{km} \mathrm{s}^{-1}}>\frac{v_{\text {sh,ad }}}{\mathrm{km} \mathrm{s}^{-1}} \simeq 650\left(\frac{n_{\mathrm{j}}}{10^{4} \mathrm{~cm}^{-3}}\right)^{\frac{2}{9}}\left(\frac{R_{\mathrm{j}}}{10^{16} \mathrm{~cm}}\right)^{\frac{2}{9}},
$$


and the jet ion density can be estimated as [9]

$$
\frac{n_{\mathrm{j}}}{\mathrm{cm}^{-3}} \approx 150\left(\frac{\dot{M}_{\mathrm{i}}}{10^{-6} \mathrm{M}_{\odot} \mathrm{yr}^{-1}}\right)\left(\frac{v_{\mathrm{j}}}{1000 \mathrm{~km} \mathrm{~s}^{-1}}\right)^{-1}\left(\frac{R_{\mathrm{j}}}{10^{16} \mathrm{~cm}}\right)^{-2}
$$

The ionized jet mass loss rate $\dot{M}_{\mathrm{i}} \propto v_{\mathrm{j}}$ is assumed to be constant along the jet and then $n_{\mathrm{j}}$ turns out to be independent of the jet velocity. By considering $\dot{M}_{\mathrm{i}}=5.6 \times 10^{-6}$ and $3.5 \times 10^{-6} \mathrm{M}_{\odot} \mathrm{yr}^{-1}$ for IRAS 16484-4603 and IRAS 16547-4247, respectively [1], and assuming $R_{\mathrm{j}}=R / 2$ we obtain the values of $n_{\mathrm{j}}$ listed in Table 1. By inserting Eq. (2) in Eq. (1) we find that $v_{\mathrm{sh}, \text { ad }} \propto R_{\mathrm{j}}^{-2 / 9}$, also listed in Table 1 . We note that $n_{\mathrm{j}}$ is a rough estimation of the jet ion density given the uncertainties in the values of $\dot{M}_{\mathrm{i}}$ and $R_{\mathrm{j}}$. Nevertheless, the derived jet density is likely lower than the typical density values of $\sim 10^{5} \mathrm{~cm}^{-3}$ in the molecular clouds where massive stars form [10] giving $\chi<1$.

\section{Cosmic Ray population}

Non-thermal electrons follow a power-law distribution $N_{\mathrm{e}}=K_{\mathrm{e}} E^{-s}$, where $s=2 \alpha+1$. The normalization factor $K_{\mathrm{e}}$ is determined from the measured synchrotron flux $S_{v}$ at a particular frequency $v$, giving

$$
\frac{K_{e}}{\mathrm{erg}^{\mathrm{s}-1} \mathrm{~cm}^{-3}} \approx 1.6 \times 10^{-9} \xi_{K}(s)\left(\frac{d}{\mathrm{kpc}}\right)^{2}\left(\frac{S_{v}}{\mathrm{mJy}}\right)\left(\frac{R}{10^{16} \mathrm{~cm}}\right)^{-3}\left(\frac{v}{\mathrm{GHz}}\right)^{\frac{s-1}{2}}\left(\frac{B_{\mathrm{s}}}{\mathrm{mG}}\right)^{-\frac{s+1}{2}},
$$

where $B_{\mathrm{S}}$ is the magnetic field in the synchrotron emitter, i.e. mostly coming from the shock downstream medium, and

$$
\xi_{K}(s) \simeq 10^{-3.55(s-2)} \frac{\Gamma\left(\frac{s+5}{4}\right) \Gamma\left(\frac{3 s-1}{12}\right) \Gamma\left(\frac{3 s+19}{12}\right)}{\Gamma\left(\frac{s+7}{4}\right)(s+1)} .
$$

The energy density in non-thermal electrons reads

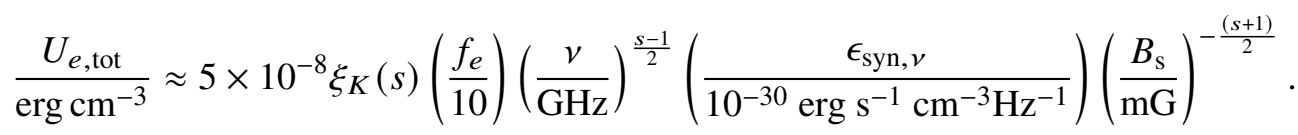

The energy density in protons is $U_{p, \text { tot }}=a U_{e, \text { tot }}$, where $a=\left(m_{\mathrm{p}} / m_{\mathrm{e}}\right)^{(s-1) / 2} f_{\mathrm{p}} / f_{\mathrm{e}}$ and $f_{e, p} \sim$ $E_{e, p \max }^{2-s} /(2-2)$ and $\left(m_{e, p} c^{2}\right)^{2-s} /(s-2)$ when $s<2$ and $>2$, respectively. The non-thermal energy density is $U_{\mathrm{nt}}=(1+a) U_{\mathrm{e} \text {,tot }}$, and then the magnetic field in equipartition with non-thermal electrons and protons is $B_{\mathrm{eq}}=\left[(1+a) U_{e, \text { tot }} 8 \pi\right]^{0.5}$. In Table 2 we list the values of $s, a$, and $B_{\mathrm{eq}}$ for all the sources in Table 1 and considering $E_{e, \max }=0.1 \mathrm{TeV}$ in $f_{e}$. (Note however that the dependence on $E_{e \text {, max }}$ is negligible.)

We define the total proton acceleration efficiency $\eta_{\mathrm{p}, \text { tot }}=U_{\mathrm{p} \text {,tot }} / U_{\text {kin }}$, where $U_{\mathrm{kin}}=n_{\mathrm{j}} v_{\mathrm{j}}^{2} / 2$ is the jet kinetic energy density. The acceleration efficiency $\eta_{\mathrm{p}}$ of protons with particular energy $E_{\mathrm{p}}$ is defined as $\eta_{p}=\eta_{p \text {,tot }} / f_{p}$. These protons can drive a current that combined with small perturbations present in the magnetic field can excite NR waves [3] when

$$
\frac{v_{\mathrm{sh}}}{\mathrm{km} \mathrm{s}^{-1}}>128\left(\frac{\eta_{p}}{0.02}\right)^{-\frac{1}{2}}\left(\frac{B_{\mathrm{j}}}{10 \mu \mathrm{G}}\right)\left(\frac{n_{\mathrm{j}}}{10^{4} \mathrm{~cm}^{-3}}\right)^{-\frac{1}{2}}
$$


Table 1: Observed and derived parameters of non-thermal lobes associated with MYSOs. From left to right we list the name of the source and the non-thermal component in the jet, the observed frequency $v$, the measured flux density $S_{v}$, the radio spectral index $\alpha$, the average linear size $R$, the synchrotron emissivity $\epsilon_{\text {synchr }}$, the lower limit on the shock velocity $v_{\text {sh,ad }}$ and the density $n_{\mathrm{j}}$.

\begin{tabular}{cccccccc}
\hline \hline Source & $\begin{array}{c}v \\
{[\mathrm{GHz}]}\end{array}$ & $\begin{array}{c}S_{v} \\
{[\mathrm{mJy}]}\end{array}$ & $\alpha$ & $\begin{array}{c}R \\
{[\mathrm{~cm}]}\end{array}$ & $\begin{array}{c}\epsilon_{\text {synchr }} \\
{\left[\mathrm{erg} \mathrm{cm}^{-3} \mathrm{~s}^{-1} \mathrm{~Hz}^{-1}\right]}\end{array}$ & $\begin{array}{c}v_{\text {sh,ad }} \\
{\left[\mathrm{km} \mathrm{s}^{-1}\right]}\end{array}$ & $\begin{array}{c}n_{\mathrm{j}} \\
{\left[\mathrm{cm}^{-3}\right]}\end{array}$ \\
\hline G339.8838 NE & 9 & 2.36 & 0.39 & $8.1 \times 10^{15}$ & $1.3 \times 10^{-28}$ & 842 & $7.9 \times 10^{4}$ \\
G343.1261 N4 & 17 & 1.80 & 0.67 & $1.0 \times 10^{16}$ & $3.2 \times 10^{-29}$ & 674 & $1.6 \times 10^{4}$ \\
G343.1261 S1 & 17 & 4.72 & 0.45 & $1.0 \times 10^{16}$ & $3.8 \times 10^{-29}$ & 633 & $9.2 \times 10^{3}$ \\
\hline
\end{tabular}

The regime dominated by Alfvén turbulence was studied by [11, 12] in the context of low mass protostars, where the jet typical velocities are $\sim 100 \mathrm{~km} \mathrm{~s}^{-1}$. The important aspect of destabilizing perturbations in resonance with CRs is that the scattering process is more efficient. However, this does not guarantee to have a strong amplification.

\section{Magnetic field amplification by Bell instabilities}

Magnetic field strengths $B_{\mathrm{s}} \approx 0.1-10 \mathrm{mG}$ are larger than the expected magnetic field in the jet termination region. In this study we consider the magnetic field amplification by the Bell instability in the non-linear case $[3,13]$. The (total) saturated magnetic field $B_{\text {sat,NR }}$ immediately upstream of the shock is estimated by considering the total proton acceleration efficiency $\eta_{p}$,tot, which gives

$$
\frac{B_{\mathrm{sat}, \mathrm{NR}}}{\mathrm{mG}} \approx 1.2\left(\frac{\eta_{p, \mathrm{tot}}}{0.1}\right)^{\frac{1}{2}}\left(\frac{n_{i}}{10^{4} \mathrm{~cm}^{-3}}\right)^{\frac{1}{2}}\left(\frac{v_{\mathrm{sh}}}{1000 \mathrm{~km} \mathrm{~s}^{-1}}\right)^{\frac{3}{2}} .
$$

In the shock downstream region, the turbulent upstream magnetic field is compressed by a factor $r_{\mathrm{B}}=\sqrt{\left(2 r^{2}+1\right) / 3} \approx 3.3$ when the shock compression ratio is $r=4$ [e.g. 7, 14]. Therefore, the amplified field downstream of the shock is $B_{\mathrm{sat}, \mathrm{d}}=r_{\mathrm{B}} B_{\mathrm{sat}, \mathrm{NR}}$. On the other hand, the magnetic field in the synchrotron emitter $B_{\mathrm{s}}$, i.e. mostly coming from the shock downstream region, is a function of the energy density in non-thermal electrons. By assuming that $B_{\mathrm{sat}, \mathrm{d}}=B_{\mathrm{s}}$ we find that $B_{\text {sat }, \mathrm{d}} / B_{\text {eq }} \sim 0.4\left(v_{\mathrm{sh}} / 1000 \mathrm{~km} \mathrm{~s}^{-1}\right)(2 /(\mathrm{s}+5))$.

\section{Gamma-ray emission}

The maximum energy of protons is determined by the amount of protons that escape from the shock upstream region [7, 8]. Given that only the most energetic protons can penetrate far upstream from the shock and amplify the magnetic field in the shock precursor, the available time to accelerate these particles is $\sim 5 / \Gamma_{\max , \mathrm{NR}}\left(E_{\mathrm{p}, \max }\right)$, where

$$
\frac{\Gamma_{\max , \mathrm{NR}}}{\mathrm{s}^{-1}} \sim 2.6 \times 10^{-5}\left(\frac{\eta_{p}}{0.02}\right)\left(\frac{v_{\mathrm{sh}}}{1000 \mathrm{~km} \mathrm{~s}^{-1}}\right)^{3}\left(\frac{n_{i}}{10^{4} \mathrm{~cm}^{-3}}\right)^{\frac{1}{2}}\left(\frac{E_{\mathrm{p}, \max }}{\mathrm{GeV}}\right)^{-1} .
$$


Table 2: From left to right we list the source names, the relativistic electrons and protons energy power-law slope $s=2 \alpha+1$, the total protons-to-electrons energy density ratio ( $a$, for the case $\left.E_{e, \max }=E_{p, \max }=1 \mathrm{TeV}\right)$, the equipartition magnetic field $\left(B_{\text {eq }}\right)$, the amplified field in the shock downstream region $\left(B_{\text {sat,NR }}\right)$, the total energy density in protons $\left(U_{p \text {,tot }}\right)$ and their acceleration efficiency $\left(\eta_{p, \text { tot }}\right)$, and the protons maximum energy $\left(E_{p, \max }\right)$.

\begin{tabular}{lccccccc}
\hline \hline Source & $s$ & $a$ & $\begin{array}{c}B_{\text {eq }} \\
{[\mathrm{mG}]}\end{array}$ & $\begin{array}{c}B_{\text {sat,NR }} \\
{[\mathrm{mG}]}\end{array}$ & $\begin{array}{c}U_{p, \text { tot }} \\
{\left[\mathrm{erg} \mathrm{cm}^{-3}\right]}\end{array}$ & $\eta_{p, \text { tot }}$ & $\begin{array}{c}E_{p, \max } \\
{[\mathrm{TeV}]}\end{array}$ \\
\hline G339.8838 NE & 1.78 & 15.06 & 14.6 & 1.63 & $3.2 \times 10^{-5}$ & 0.05 & 0.28 \\
G343.1261 N4 & 2.34 & 10.80 & 10.2 & 1.22 & $1.7 \times 10^{-5}$ & 0.13 & 0.17 \\
G343.1261 S1 & 1.89 & 63.07 & 9.93 & 1.13 & $1.5 \times 10^{-5}$ & 0.20 & 0.43 \\
\hline
\end{tabular}

is the maximum growth rate of the Bell instability driven by protons with energy $E_{p \text {,max }}$. By equating $5 / \Gamma_{\max , \mathrm{NR}}\left(E_{p, \max }\right)=R_{\mathrm{j}} / v_{\text {sh }}$ we find $[8,15]$

$$
\frac{E_{p, \max }}{\mathrm{m}_{\mathrm{p}} \mathrm{c}^{2}}= \begin{cases}70(2-s)\left(\frac{U_{p, \text { tot }}}{10^{-5} \mathrm{erg} \mathrm{cm}^{-3}}\right)\left(\frac{R_{\mathrm{j}}}{10^{16 \mathrm{~cm}}}\right)\left(\frac{n_{i}}{10^{4} \mathrm{~cm}^{-3}}\right)^{-\frac{1}{2}} & s<2 \\ 70 \log \left(\frac{E_{p, \text { max }}}{\mathrm{GeV}}\right)^{-1}\left(\frac{U_{p, \mathrm{tot}}}{10^{-5} \mathrm{erg} \mathrm{cm}^{-3}}\right)\left(\frac{R_{\mathrm{j}}}{10^{16} \mathrm{~cm}}\right)\left(\frac{n_{i}}{10^{4} \mathrm{~cm}^{-3}}\right)^{-\frac{1}{2}} & s=2 \\ {\left[70(s-2) \frac{1}{m_{p} c^{2}}\left(\frac{U_{p, \mathrm{tot}}}{10^{-5} \mathrm{erg} \mathrm{cm}^{-3}}\right)\left(\frac{R_{\mathrm{j}}}{10^{16} \mathrm{~cm}}\right)\left(\frac{n_{i}}{10^{4} \mathrm{~cm}^{-3}}\right)^{-\frac{1}{2}}\right]^{\frac{1}{s-1}}} & s>2\end{cases}
$$

Values of $E_{\mathrm{p}, \max }$ for the non-thermal lobes in our study are listed in Table 2 for the case $v_{\mathrm{sh}}=$ $1000 \mathrm{~km} \mathrm{~s}^{-1}$ and $R_{\mathrm{j}}=R / 2$. The condition $\Gamma_{\max , \mathrm{NR}}\left(E_{\mathrm{p}, \max }\right) R_{\mathrm{j}} / v_{\mathrm{sh}}>5$ was introduced by [8] for the case of SNR and where they considered the size of the spherical shock (instead of $R_{\mathrm{j}}$ ). However, [7] found a similar expression for planar non-relativistic shocks.

Electrons are not the main drivers of the turbulence and can be considered as test particles. If the Bell instability gets into the non-linear phase, we can expect to have the turbulence coherence length $\ell_{\mathrm{c}}$ limited by the Larmor radius of the protons at the maximum energy $E_{\mathrm{p}, \max }$ in the amplified field $B_{\mathrm{s}}$. If we assume that the maximum electrons energy $E_{\mathrm{e}, \max }$ exceeds $E_{\mathrm{p} \text {,max }}$, then the turbulence experienced by electrons will be in the small-scale turbulence regime and the diffusion coefficient would be $\propto D_{\text {Bohm }}\left(E_{p, \max }, B_{\mathrm{s}}\right)\left(E_{e} / E_{p, \max }\right)^{2}$. This rapid increase with the energy would limit $E_{\mathrm{e}, \max }$ to $E_{\mathrm{p}, \max }$ unless radiative losses dominate and limit it to smaller values. Then, one should expect to have $E_{\mathrm{e}, \max } \leq E_{\mathrm{p}, \max }$.

$\mathrm{TeV}$ electrons and protons can emit gamma-rays by their interaction with ambient cold protons through relativistic Bremsstrahlung and proton-proton (pp) collisions ${ }^{1}$. The distribution of relativistic protons in the jet hotspot is $N_{\mathrm{p}}=K_{\mathrm{p}} E_{\mathrm{p}}^{-s}$, where $K_{\mathrm{p}}=K_{\mathrm{e}}\left(m_{\mathrm{p}} / m_{\mathrm{e}}\right)^{(s-1) / 2}$. In Table 2 we list the values of $K_{e}$ and $K_{p}$ (with $B_{\mathrm{s}}=3.3 B_{\mathrm{sat}, \mathrm{NR}}$ ). We compute the spectral energy distribution (SED) in the one-zone model approximation. We assume that the volume of the $\gamma$-ray emitter is the same as the synchrotron emission volume and that the thermal ion density in the lobe is $4 n_{\mathrm{j}}$. We plot in Fig. 1 the SED including proton-proton collisions, synchrotron emission and relativistic Bremsstrahlung and using the formulation in [16] and [17]. We assume $E_{e, \max }=E_{p, \max }$ as it is

\footnotetext{
${ }^{1}$ Note that in the jet termination region, at $\sim 10^{4} \mathrm{AU}$ from the central protostar, the stellar photon field is very diluted and therefore the Inverse Compton scattering is not expected to be very relevant.
} 


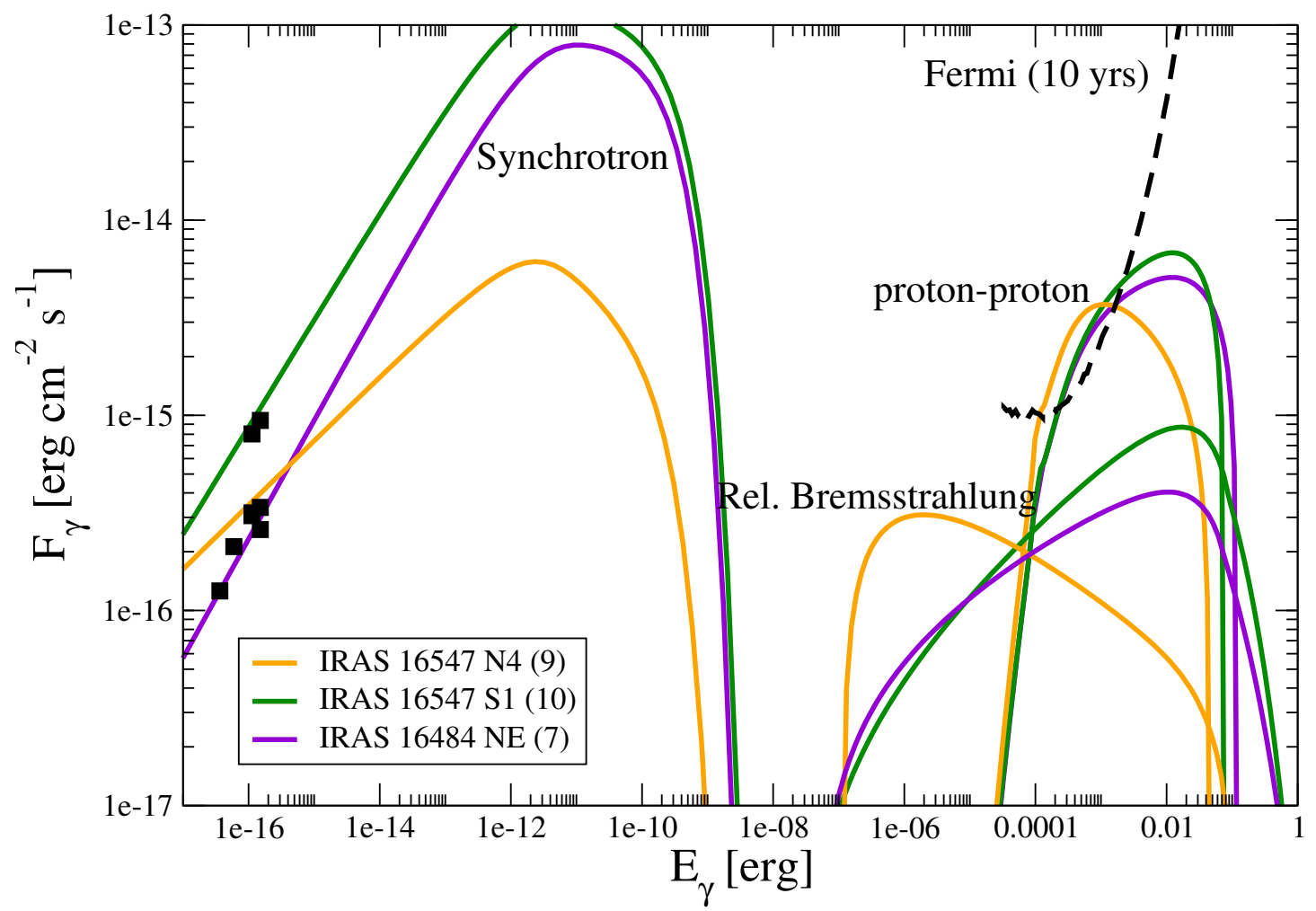

Figure 1: Spectral energy distribution for the three hotspots. Black squares indicate synchrotron data [1] and the black dashed line represent the Fermi sensitivity for 10 years of observation.

calculated in Eq. (9). We can see that the predicted emission is detectable by Fermi-LAT after 10 years of observation (with $5 \sigma$-confidence).

\section{Conclusions}

The detection of synchrotron emission from jets powered by high-mass protostars indicates that electrons are in-situ accelerated. Magnetic fields $B_{\mathrm{S}} \sim 0.3-5 \mathrm{mG}$ are needed to explain the synchrotron radio flux. These large values of $B_{\mathrm{S}}$ are difficult to explain by a simple compression in an adiabatic strong shock at the jet termination region, at $\sim 0.1 \mathrm{pc}$ from the central protostar. In the present contribution we focus on magnetic field amplification by streaming instabilities.

CR streaming instabilities can excite the NR (Bell) instability which produces perturbations at scales much smaller than $r_{\mathrm{g}}$ [3]. Bell's instability can amplify small perturbations in the magnetic field up to values much larger than the unperturbed jet magnetic field $B_{\mathrm{j}}$ when the condition in Eq. (6) is satisfied. We remark that large ion densities in a non-completely ionized protostellar jet would increase the ion-neutral collisions and Alfvén waves can be damped [18]. Conversely, Bell modes are not as heavily damped, although the maximum growth rate decreases [19].

By assuming that the large magnetic field in the synchrotron emitter $\left(B_{\mathrm{S}}\right)$ is due to amplification through the Bell's instability and fixing $v_{\mathrm{sh}}=1000 \mathrm{~km} \mathrm{~s}^{-1}$ we estimate $B_{\mathrm{s}} \sim 0.4 B_{\text {eq }}$ and the the 
proton acceleration efficiency $\eta_{p \text {,tot }} \sim 0.1$. By knowing $\eta_{p \text {,tot }}$ and $B_{\mathrm{s}}$ we estimate the maximum energy of protons accelerated in the jet reverse shock and the $\gamma$-ray emission that they produce. We find that having a density of $4 n_{\mathrm{j}}$ in the non-thermal emitter is high enough to reach detectable levels of $\gamma$ rays with Fermi in IRAS 16547 N4, IRAS 16547 S1, and IRAS 16848 NE, as we show in Fig. 1. Although there is no claim of detection of these sources by Fermi, we expect that our result will motivate a future study on the Fermi data at the location of these sources. In addition, these sources will be perfect targets for a point-source mode. We also note that mixing due to dynamical instabilities can significantly enhance the density of targets in the lobes. The very dense shell formed downstream of the radiative bow shock is unstable and fragmented in clumps with density $\sim 100-1000$ times the density of the molecular cloud. By achieving $F_{\gamma, p p} \sim 5 \times 10^{-13} \mathrm{erg} \mathrm{cm}^{-2} \mathrm{~s}^{-1}$ at $0.1 \mathrm{TeV}$, CTA will be of great importance to measure the cut-off of the spectrum. Also, the spatial resolution of CTA is expected to be better than Fermi. The detection of $\gamma$ rays from protostellar jets will open a new window to study stellar formation, as well as the efficiency of DSA in the high density $\left(n_{\mathrm{j}} \sim 10^{3}-10^{4} \mathrm{~cm}^{-3}\right)$ and low velocity $\left(v_{\mathrm{sh}} \sim 1000 \mathrm{~km} \mathrm{~s}^{-1}\right)$ regime. In particular, the detection of diffuse gamma-ray emission in molecular clouds where MYSOs are embedded will be a piece of evidence of proton acceleration in protostellar jets.

\section{Acknowledgements}

A.T.A. thanks the Czech Science Foundation under the grant GAČR 20-19854S. M.P. acknowledges funding from the INAF PRIN-SKA 2017 program 1.05.01.88.04, by the Italian Ministero dell' Istruzione, Università e Ricerca through the grant Progetti Premiali 2012-iALMA (CUP C52I13000140001). and by the project PRIN-INAF-MAIN-STREAM 2017 "Protoplanetary disks seen through the eyes of new-generation instruments". This work has been carried out thanks to the support of the OCEVU Labex (ANR-11-LABX-0060) and the A*MIDEX project (ANR-11IDEX-0001-02) funded by the "Investissements d'Avenir" French government program managed by the ANR.

\section{References}

[1] S.J.D. Purser, S.L. Lumsden, M.G. Hoare, J.S. Urquhart, N. Cunningham, C.R. Purcell et al., A search for ionized jets towards massive young stellar objects, 460 (2016) 1039 [1605.01200].

[2] K.M. Ferrière, The interstellar environment of our galaxy, Reviews of Modern Physics $\mathbf{7 3}$ (2001) 1031 [astro-ph/0106359].

[3] A.R. Bell, Turbulent amplification of magnetic field and diffusive shock acceleration of cosmic rays, 353 (2004) 550.

[4] J. Vink and J.M. Laming, On the Magnetic Fields and Particle Acceleration in Cassiopeia A, 584 (2003) 758 [astro-ph/0210669].

[5] A.T. Araudo, A.R. Bell and K.M. Blundell, Particle Acceleration and Magnetic Field Amplification in the Jets of 4C74.26, 806 (2015) 243 [1505.02210]. 
[6] A.T. Araudo, M. Padovani and A. Marcowith, Particle acceleration and magnetic field amplification in massive young stellar object jets, 504 (2021) 2405 [2102.11583].

[7] V.N. Zirakashvili and V.S. Ptuskin, Diffusive Shock Acceleration with Magnetic Amplification by Nonresonant Streaming Instability in Supernova Remnants, 678 (2008) 939 [0801 . 4488].

[8] A.R. Bell, K.M. Schure, B. Reville and G. Giacinti, Cosmic-ray acceleration and escape from supernova remnants, 431 (2013) 415 [1301.7264].

[9] A. Rodríguez-Kamenetzky, C. Carrasco-González, A. Araudo, G.E. Romero, J.M. Torrelles, L.F. Rodríguez et al., The Highly Collimated Radio Jet of HH 80-81: Structure and Nonthermal Emission, 851 (2017) 16 [1711.02554].

[10] P. Hennebelle and E. Falgarone, Turbulent molecular clouds, 20 (2012) 55 [1211.0637].

[11] M. Padovani, P. Hennebelle, A. Marcowith and K. Ferrière, Cosmic-ray acceleration in young protostars, 582 (2015) L13 [1509.06416].

[12] M. Padovani, A. Marcowith, P. Hennebelle and K. Ferrière, Protostars: Forges of cosmic rays?, 590 (2016) A8 [1602.08495].

[13] A.R. Bell, The interaction of cosmic rays and magnetized plasma, 358 (2005) 181.

[14] E. Parizot, A. Marcowith, J. Ballet and Y.A. Gallant, Observational constraints on energetic particle diffusion in young supernovae remnants: amplified magnetic field and maximum energy, 453 (2006) 387 [astro-ph/0603723].

[15] K.M. Schure and A.R. Bell, From cosmic ray source to the Galactic pool, 437 (2014) 2802 [1310.7027].

[16] G.R. Blumenthal and R.J. Gould, Bremsstrahlung, Synchrotron Radiation, and Compton Scattering of High-Energy Electrons Traversing Dilute Gases, Reviews of Modern Physics 42 (1970) 237.

[17] A.T. Araudo, G.E. Romero, V. Bosch-Ramon and J.M. Paredes, Gamma-ray emission from massive young stellar objects, 476 (2007) 1289 [0708.2955].

[18] L. Drury, P. Duffy and J.G. Kirk, Limits on diffusive shock acceleration in dense and incompletely ionised media., 309 (1996) 1002 [astro-ph/9510066].

[19] B. Reville, J.G. Kirk, P. Duffy and S. O'Sullivan, A cosmic ray current-driven instability in partially ionised media, 475 (2007) 435 [0707 . 3743]. 